\title{
La integración de las TIC en los procesos educativos y organizativos
}

\section{A integração das TIC nos processos educacionais e organizativos}

\section{The ICT's integration at educational and organizational processes}

\author{
Ana Felicitas Gargallo Castel*
}

\begin{abstract}
RESUMEN
El ámbito educativo ha incorporado gradualmente las oportunidades ofrecidas por las Tecnologías de la Información y Comunicación (TIC). Si bien este dato resulta positivo, desde el punto de vista organizativo, es necesario complementar la implantación de las TIC con una estrategia global e integradora que aporte coherencia entre los aspectos puramente tecnológicos y los modelos educativos, para alcanzar resultados superiores. El presente trabajo ofrece una revisión de la importancia de las TIC en el ámbito educativo, centrando la atención en la necesidad de ajuste estratégico en el contexto universitario, como elemento de mejora en la adaptación a las necesidades competitivas de las universidades. Para alcanzar este objetivo, se lleva a cabo una revisión de la literatura sobre las TIC y su impacto, se resumen las principales recomendaciones para su óptima utilización en el proceso de enseñanza aprendizaje y se ofrece una reflexión sobre el papel estratégico de estas herramientas.
\end{abstract}

Palabras clave: Universidad. Ventaja competitiva. TIC. Ajuste estratégico.

\section{RESUMO}

O campo da educação tem incorporado gradualmente as oportunidades oferecidas pelas Tecnologias de Informação e Comunicação (TIC). Ainda que este dado seja positivo, do ponto de vista organizacional, é necessário com-

* Universidad de Zaragoza. Departamento de Dirección y Organización de Empresas. Zaragosa, Espanha. E-mail: gargallo@unizar.es. ORCID: https://orcid.org/0000-0001-8054-8997 
plementar a implantação das TIC como uma estratégia global e integradora que traga coerência entre os aspectos puramente tecnológicos e os modelos educacionais, para alcançar melhores resultados. O presente trabalho oferece uma revisão da importância das TIC no âmbito educativo, centrando a atenção na necessidade de ajuste estratégico no contexto universitário, como elemento de melhora na adaptação às necessidades competitivas das universidades. Para alcançar este objetivo, leva-se a cabo uma revisão da literatura sobre as TIC e seu impacto, as principais recomendações são analisadas com vistas à melhor utilização no processo de ensino-aprendizagem e se oferece uma reflexão sobre o papel estratégico dessas ferramentas.

Palavras-chave: Universidade. Vantagem competitiva. TIC. Ajuste estratégico.

\begin{abstract}
In recent years, the education sector has gradually incorporated the opportunities offered by the Information Technology and Communication (ICT). While this finding is positive, from an organizational perspective, it is needed to be complemented with a comprehensive and integrated strategy that brings coherence between purely technological aspects and educational models to achieve superior results. In this paper we provide a review of the importance of ICT in education, focusing on the need of strategic alignment on the university context, as an element of improvement to adapt to the competitive needs of universities. To achieve this goal, we conducted a literature review on ICT and its impact; we summarize the main recommendations for optimal use in the teaching-learning process and offer a reflection on the strategic role of these tools.
\end{abstract}

Keywords: University. Competitive advantage. ICT. Fit.

\title{
Introducción
}

La introducción de las tecnologías de la información y la comunicación (TIC) ha facilitado un nuevo contexto tecnológico y social, no solo como un sector motor del crecimiento económico, sino como generador de importantes cambios en las empresas, en las instituciones y en la sociedad en su conjunto.

Fruto de ello, desde la literatura académica ha tenido lugar un creciente interés por el estudio de esta revolución (CHARI; DEVARAJ; DAVID, 2008; LUSE; MENNECKE, 2014). 
Al mismo tiempo, también se ha producido en los últimos años una clara generalización del uso de estas tecnologías en el ámbito educativo a lo largo de la última década y se observa que un aumento en el interés por el estudio de la gestión estratégica de los tecnologías de la información y la comunicación en este contexto (GHISLANDI, CALIDONI, FALCINELLI, SCURATI, 2008; CHER PING, 2003; GARGALLO, ESTEBAN, MARZO, 2010; SÁEZ LÓPEZ, 2010), debido sobre todo a los cambios ocurridos en el entorno, que han provocado que cada vez sea más difícil ofrecer un servicio competitivo, de calidad y adaptado a las necesidades del mercado de trabajo y de las diferentes tipologías de alumnado si no se invierte en analizar y potenciar los factores clave.

Para el caso español, desde la CRUE, a través de la Comisión Sectorial TIC se han desarrollado diversas iniciativas encaminadas a profundizar en las posibilidades de las TIC y su aplicación en el ámbito universitario, desarrollando una labor de debate y reflexión al respecto. En particular, los informes anuales UNIVERSITIC ofrecen información descriptiva sobre el estado y la evolución del uso de las TIC en las universidades españolas.

En todo caso, en el estudio de las TIC como elemento clave para la mejora competitiva y la diferenciación, debe tenerse en cuenta que las fuentes que anteriormente permitían a las empresas diferenciarse de sus competidores se han ido agotando paulatinamente, de modo que serán ahora los elementos relacionados con las capacidades organizativas los elementos diferenciadores que permiten a la empresa obtener mejores resultados que sus competidores, DE SAÁ (2000). Del mismo modo, en el ámbito universitario, será la capacidad para conjugar la implantación de las TIC con la transformación del proceso educativo, lo que permitirá a las universidades responder ante las necesidades de la sociedad actual (DE PABLOS, 2007).

\section{Marco conceptual: las TIC en la educación, necesarias pero no suficientes}

Antes de entrar en la revisión de la literatura sobre las TIC en la educación universitaria, resulta interesante recoger parte del contenido del artículo, publicado hace más de una década, en el mes de mayo de 2003 por la prestigiosa revista Hardvard Business Review, con el título "Las Tecnologías de la Información no importan" (IT Doesn't Matter) y el libro posteriormente traducido al español (CARR, 2005). En él, el autor, Nicholas G. Carr, refleja que, a pesar del aumento de las posibilidades y la sofisticación de estas tecnologías, su papel como elemento competitivo, o como fuente de ventaja competitiva ha disminuido. 
En este sentido, podría decirse que, las TIC deben verse desde el ámbito universitario como una herramienta que posibilita la consecución de mejoras en el funcionamiento interno, en los servicios prestados o en la docencia que impartimos a nuestros alumnos y que permite a su vez la entrada en nuevos segmentos de mercado que antes no podíamos alcanzar, como es el caso del aprendizaje a distancia asistido o apoyado en estas nuevas tecnologías, pero que en ningún momento se trata de una ventaja por sí misma.

Es más, en el mismo artículo de la Harvard Business Review citado con anterioridad se pone de manifiesto que "cuando un recurso -como es en este caso las tecnologías de la información y la comunicación- se convierte en un elemento esencial para competir en el mercado, puede generar unos riesgos superiores incluso a los beneficios que aporta", es decir, en ocasiones la inadecuada utilización o gestión de las distintas herramientas TIC utilizadas puede generar un alto grado de insatisfacción entre el alumnado que está recibiendo dichos servicios. Y las TIC en la educación, como en otros sectores, no sólo está sujeta a una mala gestión, sino a problemas de obsolescencia, de seguridad y confidencialidad, fallos técnicos, etc. Sería un ejemplo comparable al de la electricidad, es una fuente o un recurso "necesario", pero cuya posesión no confiere ningún tipo de ventaja competitiva; sin embargo, una breve interrupción en el suministro de la misma puede acarrear efectos desastrosos.

Los recursos de la organización pueden definirse como el conjunto de factores o activos de los que dispone o controla. La capacidad de la organización para obtener ventaja competitiva a través de sus recursos vendrá determinada por las características de esos. Desde la dirección estratégica y en concreto desde la teoría de los recursos y capacidades se defiende que para que un recurso sea fuente de ventaja competitiva, debe cumplir una serie de los requisitos, tales como: ser valioso, escaso, insustituible y difíciles de imitar (BARNEY, 1991).

Por tanto es de esperar que la simple utilización de herramientas estandarizadas, disponibles en el mercado y con características muy similares entre ellas no sea un elemento diferenciador si es utilizado por la mayoría de las universidades españolas. Por el contrario, y siguiendo con la Teoría de los Recursos y Capacidades, la organización obtendrá ventaja competitiva si consigue desarrollar recursos escasos, valiosos, difíciles de imitar, no sustituibles; activos específicos, muchas veces intangibles, que diferencian a la empresa de sus competidores. Surgen así las capacidades como las habilidades de la empresa para desarrollar adecuadamente una actividad a partir de los recursos disponibles y dentro de estas, las denominadas capacidades distintivas o competencias distintivas, a aquellas habilidades que posee la organización y que son mejores en relación con las que poseen otras entidades. 
Si bien las TIC pueden considerarse uno de los elementos externos que más ha impactado en la universidad (LLORENS, 2012), desde el momento en el que el componente "tecnológico" esté en manos de la mayoría de las universidades en igualdad de condiciones, su potencial diferenciador desaparece, por lo que el elemento que otorgará exclusividad a ese elemento recaerá en las interrelaciones de las TIC con el resto de recursos de la organización.

Será importante determinar qué factores o elementos son complementarios con la gestión de las TIC en el ámbito educativo. En concreto, en la literatura sobre dirección de empresas se ha citado la estrategia como elemento contingente, defendiendo que las inversiones en TIC serán más o menos efectivas sobre el rendimiento dependiendo de que sean consistentes con otros aspectos de la organización como es la estrategia, además de ser consistentes entre sí. Esa coherencia o ajuste debe trasladarse a todos los ámbitos de la empresa, en este caso las instituciones universitarias, siendo necesario un cambio de modelo más adaptado a las nuevas exigencias.

\section{Ajuste estratégico entre el cambio tecnológico y el educativo}

El concepto de cambio tecnológico abarca una realidad mucho más amplia que la aquí expuesta. Desde la aparición del ordenador, el desarrollo de internet, las redes sociales o las tecnologías de cloud computing son muchos los ámbitos que se han visto modificados radicalmente y el ámbito universitario es un ejemplo más de un fenómeno global (ESTEVE, 2009).

En los últimos años, son diversos los estudios que abordan el impacto de las TIC en el sector educativo (DUART; LUPIÁÑEZ, 2005; MARQUÈS, 2013; CAICEDO; ROJAS, 2014; HUERTAS; PANTOJA, 2016) ${ }^{1}$. En este punto nos encontramos con la necesidad de conjugar, por un lado la perspectiva tecnológica, centrada en nuestro caso en los cambios tecnológicos ligados a la aparición de las TIC, y por otro, la perspectiva docente y todos aquellos aspectos vinculados a la complejidad del proceso educativo, a los nuevos modelos docentes y a la propia concepción de la universidad (CABERO; MARÍN, 2014; RODRÍGUEZ CORREA, 2018).

Frente a los problemas acarreados por una visión excesivamente centrada en los aspectos puramente tecnológicos, resulta más apropiado hablar de entornos educativos tecnológicamente enriquecidos (LLORENS, 2012).

1 Para el caso español ESCALONA, GÓMEZ y ESCALONA (2017) realizan una radiografía de la presencia de las TIC en Educación a través de la producción científica. 
Las tecnologías están permitiendo que estos entornos resulten cada vez más abiertos gracias a conceptos como la accesibilidad y la ubicuidad, la computación en la nube, el m-learning, la disponibilidad de contenidos por Internet mediante streaming, las nuevas interfaces de acceso en función del perfil del usuario y de su geolocalización, la participación en las redes sociales, entre otros. Todo ello teniendo en cuenta que el modelo de aprendizaje en todo momento debe apoyarse en el papel activo del estudiante como actor principal en un entorno colaborativo e interactivo, que refuerce el aprendizaje autónomo.

En el caso que nos ocupa, deberán ser las propias universidades y los mismos docentes los que sean capaces de utilizar las herramientas disponibles a su alcance para lograr entornos de aprendizaje que se adapten a cada uno de los contextos y que contribuyan a facilitar los sistemas de enseñanza-aprendizaje. Por tanto, resulta crucial el apoyo y la apuesta institucional de las universidades, así como la actitud de los docentes y su predisposición al cambio y a la mejora continua apoyada en las nuevas tecnologías. Tal y como se ha puesto de manifiesto en abundantes trabajos, el papel de los recursos humanos en el aprovechamiento de las TIC es fundamental (BHARADWAJ, 2000; BRESNAHAN; BRYNJOLFSSON; HITT, 2002; GARGALLO; GALVE, 2012). En el ámbito universitario en concreto, para que las TIC se integren en el proceso de enseñanza aprendizaje, es imprescindible que los profesores sean partícipes de la reflexión y el debate del modelo educativo (SÁEZ, 2010; SOMEKH, 2007).

A este respecto, entre las principales barreras para la integración de las TIC en la educación se han identificado aspectos relacionados con la capacitación, el conocimiento y las creencias que tienen los docentes sobre las TIC y su relación con las prácticas docentes (ERTMER; OTTENBREIT-LEFTWICH; SADIK; SENDURUR; SENDURUR, 2012). Por ello resulta clave la sensibilización y formación de los docentes para que su uso no se limite a su función informativa y de tratamiento de la información (MORALES, TRUJILLO, RASO, 2015; Mirete, 2016). Pero además, deben acometerse de manera conjunta cuestiones como las siguientes:

- La definición clara del modelo pedagógico a partir del cual identificar las necesidades y las tecnologías más apropiadas.

- La identificación y generalización de las buenas prácticas apoyadas en las TIC realizadas tanto dentro como fuera de la institución (benchmarking).

- La existencia de una clara estrategia institucional que provea un adecuado desarrollo de las TIC en la enseñanza

- La promoción de entornos interactivos y colaborativos que favorezcan los procesos de enseñanza-aprendizaje. 
Todo esto permitirá optimizar el uso de las TIC para el aprendizaje y con ello su aportación a las exigencias del nuevo marco común de referencia en el Espacio Europeo de Educación Superior (ESEE). Tal y como ha venido a acuñarse, debe pasarse de las Tecnologías de la Información y la Comunicación (TIC) a las Tecnologías del Aprendizaje y el Conocimiento (TAC).

En ese contexto, la Comisión Sectorial de Tecnologías de la Información y las Comunicaciones de la CRUE, con la colaboración de la Secretaría General de Universidades del Ministerio de Educación elabora desde el año 2007 los informes UNIVESITIC. Con esta publicación desean ofrecer una radiografía exhaustiva del estado del Sistema Universitario Español en cuanto a estas tecnologías. El informe UNIVESITIC (UCEDA; PÍRIZ, 2012) recoge cuatro grandes grupos de universidades atendiendo a su grado de integración de las TIC en los procesos educativos y organizativos, según la propuesta llevada a cabo en el estudio de PLS RAMBOLL MANAGEMENT (2004). Atendiendo a esta clasificación, puede distinguirse entre las universidades punteras, las universidades cooperantes, las universidades autosuficientes y las universidades escépticas, tal y como se recoge a continuación:

a) Universidades punteras: En este grupo, también conocido como frontrunners, se recogen aquellas universidades en las que la integración de las TIC dentro de los ámbitos educativos y organizacionales resulta fundamental, tanto como parte de la práctica docente habitual, como para la formación continua académica u otra formación continua adicional. Para ello utiliza fundamentalmente los cursos e-learning. El área TIC presenta un desarrollo claro, procedente de las propias universidades, mediante estrategias explícitas que demuestran el interés prioritario desde el nivel directivo. Se trata de instituciones con tamaños muy diferentes y en las que destaca su cooperación con otras universidades y con otros agentes tales como empresas privadas.

b) Estas universidades representan aproximadamente el 16\% del total y probable incrementen su ritmo de crecimiento y su liderazgo en el entorno europeo dentro de los próximos años, reforzando sus lazos de cooperación con otros actores públicos y privados a través del incremento de su oferta de actividades y de cursos de e-learning.

c) Universidades cooperantes: Las universidades cooperantes o cooperating universities, presentan un nivel de integración de las TIC considerable, especialmente en el ámbito organizacional. Sin embargo, se encuentran todavía alejadas de las universidades punteras. Estas instituciones han logrado una integración avanzada de las TIC en las actividades docentes habituales, si bien dicho nivel es menor en 
el ámbito de la formación continua académica y la complementaria. En cuanto a las actitudes hacia las TIC, estas son positivas tanto por parte de la dirección como de los alumnos. Sin embargo existe todavía una parte importante de profesores que se muestran escépticos. Estas universidades presentan diversas relaciones de cooperación con otras instituciones educativas, y sus procesos de integración de las TIC suelen estar financiados parcialmente con fondos públicos. A nivel estratégico se recoge la importancia de las TIC, pero no como una prioridad, lo que explica que se encuentren en un estadio inferior al alcanzado por las universidades punteras y que esta situación se mantenga en los próximos años. En su evolución, probablemente se enfoquen hacia el desarrollo de las habilidades tecnológicas del profesorado, del incremento de su oferta de cursos e-learning y de lazos con otras instituciones educativas, aunque no así con otras entidades privadas. En cuanto a su importancia relativa, se estima que representan actualmente un 33\% sobre el total de universidades.

d) Universidades autosuficientes: Presentan un perfil similar a las universidades cooperantes en relación a la integración de las TIC y a las actitudes positivas hacia las nuevas tecnologías. Sin embargo, cuentan con una mayor proporción de profesores escépticos ante la integración de las TIC, así como una menor propensión al establecimiento de lazos de cooperación con otros agentes. Se puede reseñar que es el grupo de mayor tamaño y que una gran proporción de este grupo (28\%) son instituciones de gran tamaño, con más de 20.000 alumnos. En el futuro estas universidades probablemente logren una integración de las TIC desde un punto de vista interno, aunque su desarrollo hacia el exterior y el establecimiento de lazos de cooperación parece algo más difícil. Estas representan aproximadamente el 36\% sobre el total.

e) Universidades escépticas: Las universidades escépticas o self-sufficient universities presentan un retraso importante en la mayor parte de las dimensiones revisadas. Tanto en el grado de integración de las TIC en los procesos docentes, como en la participación en redes de cooperación, e igualmente en el escepticismo que muestran el profesorado y la dirección. De forma coherente, la financiación de las TIC en estas organizaciones depende del gobierno y de las administraciones públicas. Los autores estiman que representan un 15\% del total, siendo el grupo menos numeroso. Además, las universidades de pequeño tamaño, con menos de 10.000 alumnos representan casi la mitad del mismo. Las previsiones para este colectivo apuntan hacia un lento desarrollo del área TIC centrado fundamentalmente en la formación de profesores y 
alumnos y en el desarrollo de las infraestructuras TIC necesarias. Su importancia relativa es del $36 \%$.

Las universidades clasificadas como punteras representan el $16 \%$ del total, cifra muy alejada de los valores superiores al $30 \%$ en el caso de las universidades cooperantes y autosuficientes ( $33 \%$ y $36 \%$, respectivamente). Esto confirma su ventaja competitiva frente a sus competidores y nos indica desde una perspectiva estratégica hacia donde deben caminar nuestras universidades, buscando un compromiso claro desde los órganos directivos que permita la integración total de las TIC en las en todos los ámbitos.

Este es un elemento común a cualquier proceso de cambio, dado que "es la alta dirección quien tienen la posición y el potencial para iniciar e implantar el cambio estratégico" (TUSHMAN; ROMANELLI, 1985: 209). Igualmente, resulta necesaria la implicación de la alta dirección en las decisiones de implementación e integración de las TIC en cualquier ámbito, pues requieren de recursos y cambios organizativos cuya disponibilidad dependerá de dicho apoyo (PREMKUMAR; ROBERTS, 1999; FORTH; MASON, 2004). A este respecto, WEILL y ARAL (2006) citan la implicación constante de la dirección como uno de los factores característicos de las empresas que obtienen mayores resultados de las TIC. Además, estos y otros autores subrayan que resulta indispensable la implicación y habilidades del personal, en este caso de los estudiantes y del profesorado, lo que se ve nuevamente reflejado en la clasificación de PLS RAMBOLL MANAGEMENT (2004) para el caso de las universidades con mayor grado de integración de las TIC. En el ámbito universitario, LEWIS, AGARWAL y SAMBAMURTHY (2003) examinan el uso de la web por los estudiantes y los profesores de una gran universidad y sus resultados sugieren que el compromiso de la alta dirección con la tecnología y la capacidad innovadora del personal influyen positivamente en su percepción sobre la utilidad y la facilidad de uso de las TIC. Como indica SÁEZ LÓPEZ (2010), para el uso efectivo de las TIC es necesaria una actitud positiva de los docentes y un adecuado nivel de formación y manejo de estas utilidades.

Por otra parte, se comprueba que la capacidad para cooperar con otras universidades y con otros agentes y empresas públicas o privadas se convierte en un elemento diferencial (PATRAKOSOL; OLSON, 2007). Como se ha demostrado en el ámbito de la empresa, las TIC facilitan la comunicación y el acceso a la información, ofrecen oportunidades para la reducción de los costes de transacción y de coordinación al facilitar la cooperación entre agentes (BARUA; RAVINDRAN; WHINSTON, 2007; HEMPELL; ZWICK, 2008; SALAS, 2001). A ese respecto, autores como ARVANITIS y HOLLENSTEIN (2001) o LAL (2002) obtienen un impacto positivo de la colaboración con otras empresas o con instituciones de investigación en la adopción e inversión en TIC. 
Por todo ello, no resulta extraño que las universidades más avanzadas en el aprovechamiento de las TIC también desarrollen abundantes relaciones de cooperación. A nivel europeo, iniciativas como los Campus de Excelencia Internacional (MINISTERIO DE EDUCACIÓN, CULTURA Y DEPORTE, 2016; DELGADO, 2017) ofrecen una muestra de los esfuerzos realizados en dicha dirección.

Finalmente, como muestran los resultados, cuestiones como el tamaño no suponen una limitación, por lo que tanto las universidades con mayor relevancia y dimensión, como aquellas de menor tamaño pueden alcanzar niveles de integración competitivos.

\section{Tendencias en la aplicación de las TIC}

Una vez vistas en líneas generales las características de las universidades más activas en la integración de las TIC, en este apartado se incorporan las recomendaciones que deben ser tenidas en cuenta para el correcto aprovechamiento del vasto conjunto de opciones ofrecidas por las TIC. Las tendencias en la aplicación de las TIC para la docencia universitaria pasan por tener en cuenta las siguientes indicaciones (LLORENS, 2012; PARDO; CAMARILLO; JIMÉNEZ, 2016):

- Avanzar hacia infraestructuras tecnológicas flexible, sostenible y escalable.

- Fomentar la cultura digital a todos los niveles.

- Diseñar el modelo pedagógico antes que el modelo tecnológico

- Sustentar los avances sostenibles y consecuentes.

- Gestionar un almacenamiento en constante crecimiento, hacia un modelo basado en la nube (cloud computing).

- Utilizar modelos y arquitecturas abiertas que faciliten la adopción de nuevas plataformas de m-learning y herramientas sociales.

- Fomentar el diálogo en red y los grupos de trabajo de docentes, una tendencia que debe estar encaminada hacia la creación de comunidades de aprendizaje.

- Garantizar una gestión eficiente del cambio a través de la programación en las universidades de la innovación.

- Proporcionar una actitud positiva y constructiva frente al cambio para evitar la brecha digital dentro de la propia universidad, facilitando la transición a nuevos modelos de aprendizaje. 
- Fortalecer la movilidad virtual y los aspectos interculturales como un valor añadido del uso de las TIC y como complemento de otros tipos de movilidad tradicionales (Erasmus, intercambios y estancias).

- Potenciar la creación de materiales docentes en abierto (bajo licencias creative commons) como alternativa a la proliferación de materiales en la red.

- Proporcionar al estudiante formación para las nuevas competencias que deben ser adquiridas para estas modalidades de aprendizaje basadas en TIC.

- Acercar al estudiante, como usuario final, mayor información sobre los servicios TIC universitarios para optimizar su potencialidad en el proceso enseñanza - aprendizaje.

- Acometer una redefinición del puesto de trabajo del estudiante, ya que las características de las aulas de teoría y prácticas empiezan a difuminarse.

- Fomentar la implicación de los estudiantes (antes, durante y como egresados) en todo el proceso de definición del modelo y estrategia educativa.

- Incorporar al análisis estratégico la creciente importancia del aprendizaje a lo largo de la vida (LLL o Life Long Learning).

\section{Conclusiones}

Tras las diversas reflexiones sobre la importancia de las TIC como factor estratégico dentro de la enseñanza universitaria, cabe destacar cómo las TIC por si solas no constituyen una fuente de ventaja competitiva sostenible, sino que deben estar muy ligadas a aspectos como la estrategia de la empresa, la cultura, el modelo educativo elegido o los recursos humanos de la misma. En todo momento, los equipos directivos de los distintos centros educativos deben revisar este ajuste y estar muy pendientes de los cambios en el entorno, lo que obliga a concienciarse de la necesidad y la importancia de un análisis profundo y continuo. Esta constante atención a los cambios resulta en estos momentos todavía más importante si cabe. Tanto la actual convergencia con Europa en un modelo educativo centrado en el estudiante y encaminado al desarrollo de competencias, como el contexto económico y social actual obligan a que conceptos como la flexibilidad, la innovación, la adaptabilidad o la interconectividad estén presentes en todas las organizaciones.

Además, desde el punto de vista del modelo de enseñanza, debe seguir avanzándose en los marcos colaborativos, encaminados a aprovechar las opor- 
tunidades que brindan las redes y alianzas de colaboración entre universidades para el trabajo conjunto.

\section{REFERENCIAS}

ARVANITIS, S.; HOLLENSTEIN, H. The Determinants of the Adoption of Advanced Manufacturing Technology: An Empirical Analysis Based on Firm-Level Data for Swiss Manufacturing. Economics of Innovation and New Technology, v. 10, n. 5, p. 377-414, 2001.

BARNEY, J. Firm resources and sustained competitive advantage. Journal of Management, v. 17, n. 1, p. 99-120, 1991.

BARUA, A.; RAVINDRAN, S.; WHINSTON, A. B. Enabling information sharing within organizations. Information Technology Management, v. 8, p. 31-45, 2007.

BHARADWAJ, A. S. A resource-based perspective on information technology capability and firm performance: an empirical investigation. MIS Quarterly, v. 24, n. 1, p. 169-197, 2000.

BRESNAHAN, T. F.; BRYNJOLFSSON, E.; HITT, L. M. Information technology, workplace organization, and the demand for skilled labor: firm-level evidence. The Quarterly Journal of Economics, v. 117, n. 1, p. 339-376, 2002.

CAICEDO, A. M.; ROJAS, T. Creencias, conocimientos y usos de las TIC de los profesores universitarios. Educ., v. 17, n. 3, p. 517-533, 2014.

CARR, N. G. Las tecnologías de la información ¿Son realmente una ventaja competitiva? Ediciones Urano SA, Barcelona: Empresa Activa, 2005.

CABERO, J.; MARÍN, V. Miradas sobre la formación del profesorado en tecnologías de información y comunicación TIC. Revista Venezolana de Información de Tecnología y Conocimiento, v. 2, p. 11-24, 2014.

CHARI, M. D. R.; DEVARAJ, S.; DAVID, P. The impact of information technology investments and diversification strategies on firm performance. Management Science, v. 54, n. 1, p. 224-234, 2008.

CHER PING, L. Information and Communication Technologies ICT Addressing the Challenges of Economics Education: To Be or Not To Be? International Review of Economics Education, v. 1, n. 2, p. 25-54, 2003.

DE PABLOS, J. El cambio metodológico en el Espacio Europeo de Educación Superior y el papel de las tecnologías de la información y la comunicación. RIED. Revista Iberoamericana de Educación a Distancia, 2007, v. 10, n. 2. 
DE SAÁ, P. Valor estratégico de los recursos humanos según la visión de la empresa basada en los recursos. Revista Europea de Dirección y Economía de Empresa, v. 9, n. 2, p. $97-116.2000$.

DELGADO, L. Campus de Excelencia Internacional. Hacia una reforma estructural del Sistema Universitario Español. La Cuestión Universitaria, v. 9, p. 46-59, 2017.

DUART, J. M.; LUPIÁÑEZ, F. E-strategias en la introducción y uso de las TIC en la universidad. Revista de Universidad y Sociedad del Conocimiento, v. 2, n. 1, p. 5-31, 2005.

ERTMER, P. A.; OTTENBREIT-LEFTWICH, A. T.; SADIK, O.; SENDURUR, E.; SENDURUR, P. Teacher beliefs and technology integration practices: A critical relationship. Computers \& Education, v. 59, p. 423-435, 2012.

ESCALONA FERNANDEZ, J.; GÓMEZ MARTÍN, P.; ESCALONA FERNÁNDEZ, I. Las TIC en la educación española a través de las publicaciones periódicas: un análisis bibliométrico. Pixel-Bit. Revista de Medios y Educación, v. 51, p. 21-36, 2017.

ESTEVE, F. Bolonia y las TIC: de la docencia 1.0 al aprendizaje 2.0. La Cuestión Universitaria, v. 5, p. 58-67, 2009.

LUSE, A.; MENNECKE, B. IT can matter: co-evolution fostering IT competitive advantage. Management Research Review, v. 37, n. 6, p. 574-588, 2014.

FORTH, J.; MASON, G. Information and communication technology ICT adoption and utilisation, skill constraints and firm- level performance: evidence from UK benchmarking surveys. National Institute of Economic and Social Research (NIESR), Discussion Paper, n. 234, London, 2004.

GARGALLO, A.; GALVE, C. The Impact of ICT on Productivity: The Moderating Role of Worker Quality and Quality Strategy. En: SUN, HongYi (Ed.). Management of Technological Innovation in Developing and Developed Countries. Editorial INTECH, 2010.

GARGALLO, A.; ESTEBAN, L.; MARZO, M. Are new technologies influencing the academic results achieved by students? An exploratory study. Turkish Online Journal of Distance Education-TOJDE, v. 11, n. 3, 2010.

GHISLANDI, P.; CALIDONI, P.; FALCINELLI, F.; SCURATI, C. e-university: A cross-case study in four Italian universities. British Journal of Educational Technology, v. 39, n. 3, p. 443-455, 2008.

HEMPELL, T.; ZWICK, T. New technology, work organisation, and innovation. Economics of Innovation and New Technology, v. 17, n. 4, p. 331-354, 2008.

HUERTAS, A.; PANTOJA, A. Efectos de un programa educativo basado en el uso de las TIC sobre el rendimiento académico y la motivación del alumnado en la asignatura de tecnología de educación secundaria. Educación XX1, v. 19, n. 2, p. 229-250, 2016.

LAL, K. E-business and manufacturing sector: a study of small and medium-sized enterprises in India. Research Policy, v. 31, n. 7, p. 1199-1211, 2002. 
LEWIS, W.; AGARWAL, R.; SAMBAMURTHY, V. Sources of influence on beliefs about information technology use: an empirical study of knowledge workers. MIS Quarterly, v. 27, n. 4, p. 657-678, 2003.

LLORENS, F. (Coord.). Tendencias TIC para el Apoyo a la Docencia Universitaria. Informe CRUE, 2012.

MARQUÈS, P. R. Impacto de las TIC en la educación: Funciones y limitaciones. $3 c$ TIC: cuadernos de desarrollo aplicados a las TIC, v. 2, n. 1, p. 1-15, 2013.

MINISTERIO DE EDUCACIÓN, CULTURA Y DEPORTE. Programa Campus de Excelencia Internacional, 2016. Recuperado de: $<$ http://www.mecd.gob.es/servicios-al-ciudadano-mecd/catalogo/general/educacion/0/cei/cei-2009.html>. Acceso en: 29 dic. 2018 .

MIRETE, A. B. El profesorado universitario y las TIC. Análisis de su competencia digital. ENSAYOS, Revista de la Facultad de Educación de Albacete, v. 31, n. 1, p. 133-147, 2016.

MORALES, M.; TRUJILLO, J. M.; RASO, F. Percepciones acerca de la integración de las TIC en el proceso de enseñanza-aprendizaje de la universidad. Pixel-Bit. Revista de Medios y Educación, v. 46, p. 103-117, 2015.

PATRAKOSOL, B.; OLSON, D. L. How interfirm collaboration benefits IT innovation. Information \& Management, v. 44, n. 1, p. 53-62, 2007.

PLS RAMBOLL MANAGEMENT. Studies in the Context of the E-learning Initiative: Virtual Models of European Universities Lot 1. Draft Final Report to the UE25 Commission, DG Education \& Culture, 2004.

PRADO, A.; CAMARILLO, J.; JIMÉNEZ, T. TIC 360. Una visión completa de las TIC en el sistema Universitario Español para una evolución necesaria. Conferencia de Rectores de las Universidades Españolas (CRUE), 2016.

PREMKUMAR, G.; ROBERTS, M. Adoption of new information technologies in rural small businesses. Omega, International Journal of Management Science, v. 27, n. 4, p. 467-484, 1999.

RODRÍGUEZ CORREA, M. La incorporación de las tecnologías de la información y la comunicación en las universidades: experiencias y prácticas. Tendencias Pedagógicas, v. 31, p. 275-288, 2018.

SÁEZ LÓPEZ, J. M. Utilización de las TIC en el proceso de enseñanza aprendizaje, valorando la incidencia real de las tecnologías en la práctica docente. Docencia e Investigación: Revista de la Escuela Universitaria de Magisterio de Toledo, v. 20, n.10 versión digital, p. 183-204, 2010.

SALAS, V. La dimensión de la empresa en la economía de la información. Papeles de Economía Española, v. 89-90, p. 2-17, 2001. 
SOMEKH, B. Pedagogy and learning with ICT. Researching the art of innovation. London, Routledge, 2007.

TUSHMAN, M.; ROMANELLI, E. Organizational Evolution: A Metamorphosis Model of Convergence and Reorientation. Research in Organizational Behavior, v. 7, p. 171$222,1985$.

UCEDA, J.; PÍRIZ, S. UNIVERSITIC 2012. Descripción, gestión y gobierno de las TI en el Sistema Universitario Español. Conferencia de Rectores de las Universidades Españolas, 2012.

WEILL, P.; ARAL, S. Generating premium returns on your IT investments. Sloan Management Review, v. 47, n. 2, p. 39-48, 2006.

Texto recibido el 03 de enero de 2018. Texto aprobado el 05 de febrero de 2018. 
